\title{
Open-charm meson spectroscopy
}

\author{
J. Vijande, F. Fernández, A. Valcarce \\ Grupo de Física Nuclear and IUFFyM, \\ Universidad de Salamanca, E-37008 Salamanca, Spain
}

\begin{abstract}
We present a theoretical framework that accounts for the new $D_{J}$ and $D_{s J}$ mesons measured in the open-charm sector. These resonances are properly described if considered as a mixture of conventional $P$-wave quark-antiquark states and four-quark components. The narrowest states are basically $P$-wave quark-antiquark mesons, while the dominantly four-quark states are shifted above the corresponding two-meson threshold, being broad resonances. We study the electromagnetic decay widths as basic tools to scrutiny their nature. The proposed explanation incorporates in a natural way the most recently discovered mesons in charmonium spectroscopy.
\end{abstract}

Keywords: Charm mesons, Charm-strange mesons, quark models.

Pacs: 14.40.Lb, 14.40.Ev, 12.39.Pn. 


\section{INTRODUCTION}

During the last few years, heavy meson spectroscopy is living a continuous excitation due to the discovery of several new charmed mesons. Two years ago BABAR Collaboration at the Standford Linear Accelerator Center (SLAC) reported the observation of a charmstrange state, the $D_{s J}^{*}(2317)[1]$. It was confirmed by CLEO Collaboration at the Cornell Electron Storage Ring [2] and also by Belle Collaboration at KEK [3]. Besides, BABAR had also pointed out to the existence of another charm-strange meson, the $D_{s J}(2460)$ [1]. This resonance was measured by CLEO [2] and confirmed by Belle [3]. Belle results [3] are consistent with the spin-parity assignments of $J^{P}=0^{+}$for the $D_{s J}^{*}(2317)$ and $J^{P}=$ $1^{+}$for the $D_{s J}(2460)$. Thus, these two states are definitively well established, confirmed independently by different experiments. They present unexpected properties, quite different from those predicted by quark potential models. If they would correspond to standard $P$-wave mesons made of a charm quark, $c$, and a strange antiquark, $\bar{s}$, their masses would be larger [4], around $2.48 \mathrm{GeV}$ for the $D_{s J}^{*}(2317)$ and $2.55 \mathrm{GeV}$ for the $D_{s J}(2460)$. They would be therefore above the $D K$ and $D^{*} K$ thresholds, respectively, being broad resonances. However the states observed by BABAR and CLEO are very narrow, $\Gamma<4.6 \mathrm{MeV}$ for the $D_{s J}^{*}(2317)$ and $\Gamma<5.5 \mathrm{MeV}$ for the $D_{s J}(2460)$.

The intriguing situation of the charm-strange mesons has been translated to the nonstrange sector with the Belle observation [5] of a nonstrange broad scalar resonance, $D_{0}^{*}$, with a mass of $2308 \pm 17 \pm 15 \pm 28 \mathrm{MeV} / \mathrm{c}^{2}$ and a width $\Gamma=276 \pm 21 \pm 18 \pm 60 \mathrm{MeV}$. A state with similar properties has been suggested by FOCUS Collaboration at Fermilab [6] during the measurement of masses and widths of excited charm mesons $D_{2}^{*}$. They found significant evidence for a broad excess parametrized as an $S$-wave resonance. Without being able to clearly distinguish the origin of this broad excess, they conclude that their results are in agreement with the Belle results [5]. This state generates for the open-charm nonstrange mesons a very similar problem to the one arising in the strange sector with the $D_{s J}^{*}(2317)$. If the $D_{0}^{*}(2308)$ would correspond to a standard $P$-wave meson made of a charm quark, $c$, and a light antiquark, $\bar{n}$, its mass would have to be larger, around $2.46 \mathrm{GeV}$. In this case, the quark potential models prediction and the measured resonance are both above the $D \pi$ threshold, the large width observed being expected although not its low mass.

The last step in this series of discoveries has been the observation of a new charm-strange meson, $D_{s J}$, announced by SELEX Collaboration at Fermilab [7] with a mass of $2632.5 \pm 1.7$ $\mathrm{MeV} / \mathrm{c}^{2}$ and a small width, $\Gamma<17 \mathrm{MeV}$. However, up to now no other experiment has been able to confirm the existence of this resonance [8].

There have been many theoretical interpretations for the masses and widths of the new resonances, but most part of them have been devoted to explain the strange states. Ref. 9] made use of a unitarized meson model, the existence of a quasi-bound $c \bar{s}$ state due to the coupling with the nearby $S$-wave $D K$ threshold. The coupling to the $D K$ channel within a QCD string model an a chiral Lagrangian was used in Ref. 10]. Ref. 11 proposed a $c \bar{s}$ structure with modified non-central forces. Ref. [12] combined HQET with a chiral effective Lagrangian to interpret these states as the missing $j=1 / 2$ member of the $c \bar{s} L=1$ ground state multiplet, being $j$ the angular momentum of the strange quark. The smaller

mass of the $D_{s J}^{*}(2317)$ is attributed in Ref. [13] to a coupled channel effect. Finally more involved qualitative solutions like $D K$-molecules [14], $D_{s} \pi$-atoms [15], four-quark states [16, 17] and their combination with $q \bar{q}$ states [18] have been invoked to explain why the new states have a nature so different from canonical $q \bar{q}$ mesons. Although the nonstrange 
partners of the $D_{s J}$ 's have received much less attention, as discussed above, they present similar spectroscopic properties that should be acknowledge altogether with those of the strange states in any reliable model.

The difficulties to identify the $D_{J}$ and $D_{s J}$ states with conventional $c \bar{q}$ mesons are rather similar to those appearing in the light-scalar meson sector [19] and may be indicating that other configurations are playing a role, as could be for example four-quark contributions. $q \bar{q}$ states are more easily identified with physical hadrons when virtual quark loops are not important. This is the case of the pseudoscalar and vector mesons, mainly due to the $P$-wave nature of this hadronic dressing. On the contrary, in the scalar sector it is the $q \bar{q}$ pair the one in a $P$-wave state, whereas quark loops may be in a $S$-wave. In this case the intermediate hadronic states that are created may play a crucial role in the composition of the resonance, in other words unquenching may be important. The vicinity of these components to the lightest $q \bar{q}$ state implies that they have to be considered. This has been shown as a possible interpretation of the low-lying light-scalar mesons, where the coupling of the scalar $q \bar{q}$ nonet to the lightest $q q \bar{q} \bar{q}$ configurations allows for an almost one-to-one correspondence between theoretical states and experiment [20].

In this work we pretend to explore the same ideas for the understanding of the properties of the $D_{J}$ and $D_{s J}$ meson states. For this purpose, in the next section we will present our calculating scheme and the basic ingredients of the constituent quark model used. Section III will be devoted to present and discuss our results in connection with those obtained for the light-scalar mesons. Finally, in Sec. IV we will resume the most important conclusions of our work.

\section{CALCULATING FRAMEWORK}

In non-relativistic quark models gluon degrees of freedom are frozen and therefore the wave function of a zero baryon number $(B=0)$ hadron may be written as

$$
|\mathrm{B}=0\rangle=\Omega_{1}|q \bar{q}\rangle+\Omega_{2}|q q \bar{q} \bar{q}\rangle+\ldots
$$

where $q$ stands for quark degrees of freedom and the coefficients $\Omega_{i}$ take into account the mixing of four-quark and $q \bar{q}$ states. $|\mathrm{B}=0\rangle$ systems could then be described in terms of a hamiltonian

$$
H=H_{0}+H_{1} \text { being } \quad H_{0}=\left(\begin{array}{cc}
H_{q \bar{q}} & 0 \\
0 & H_{q q \bar{q} \bar{q}}
\end{array}\right) \quad H_{1}=\left(\begin{array}{cc}
0 & V_{q \bar{q} \leftrightarrow q q \bar{q} \bar{q}} \\
V_{q \bar{q} \leftrightarrow q q \bar{q} \bar{q}} & 0
\end{array}\right),
$$

where $H_{0}$ is a constituent quark model hamiltonian described below and $H_{1}$, that takes into account the mixing between $q \bar{q}$ and $q q \bar{q} \bar{q}$ configurations, includes the annihilation operator of a quark-antiquark pair into the vacuum. This operator could be described using the ${ }^{3} P_{0}$ model, however, since this model depends on the vertex parameter, we prefer in a first approximation to parametrize this coefficient by looking to the quark pair that is annihilated and not to the spectator quarks that will form the final $q \bar{q}$ state. Therefore we have taken $V_{q \bar{q} \leftrightarrow q q \bar{q} \bar{q}}=\gamma$. If this coupling is weak enough one can solve independently the eigenproblem for the hamiltonians $H_{q \bar{q}}$ and $H_{q q \bar{q} \bar{q}}$, treating $H_{1}$ perturbatively. To ensure that the perturbative treatment is justified, $\gamma$ cannot take all possible values, being restricted to $\left|\gamma /\left(E_{J^{P C}}^{n}-E_{J^{P C}}^{n+1}\right)\right|^{2} \leq 1$. This restriction will limit the energy range of the mixed states once the unmixed energies are calculated. The two-body problem has been solved exactly by 
means of the Numerov algorithm [21]. The four-body problem has been solved by means of a variational method using the most general combination of gaussians as trial wave functions 22, 23]. In particular, the so-called mixed terms (mixing the various Jacobi coordinates) that are known to have a great influence in the light quark case have been considered.

Although the constituent quark model used is described in Ref. [21], let us outline here its basic ingredients. Since the origin of the quark model hadrons have been considered to be built by constituent (massive) quarks. Nowadays it is widely recognized that the constituent quark mass of light quarks appears because of the spontaneous breaking of the original chiral symmetry of the QCD Lagrangian, what gives rise to boson-exchange interactions between quarks. The different terms of the potential contain central and tensor or central and spinorbit contributions that will be grouped for consistency. Therefore, the chiral part of the quark-quark interaction can be resumed as follows,

$$
V_{\chi}\left(\vec{r}_{i j}\right)=V_{\chi}^{C}\left(\vec{r}_{i j}\right)+V_{\chi}^{T}\left(\vec{r}_{i j}\right)+V_{\chi}^{S O}\left(\vec{r}_{i j}\right),
$$

where $C$ stands for central, $T$ for tensor, and $S O$ for spin-orbit potentials. The central part of the quark-quark meson-exchange potentials are given by:

$$
V_{\chi}^{C}\left(\vec{r}_{i j}\right)=V_{\pi}^{C}\left(\vec{r}_{i j}\right)+V_{\sigma}^{C}\left(\vec{r}_{i j}\right)+V_{K}^{C}\left(\vec{r}_{i j}\right)+V_{\eta}^{C}\left(\vec{r}_{i j}\right),
$$

each contribution given by,

$$
\begin{aligned}
V_{\pi}^{C}\left(\vec{r}_{i j}\right) & =\frac{g_{c h}^{2}}{4 \pi} \frac{m_{\pi}^{2}}{12 m_{i} m_{j}} \frac{\Lambda_{\pi}^{2}}{\Lambda_{\pi}^{2}-m_{\pi}^{2}} m_{\pi}\left[Y\left(m_{\pi} r_{i j}\right)-\frac{\Lambda_{\pi}^{3}}{m_{\pi}^{3}} Y\left(\Lambda_{\pi} r_{i j}\right)\right]\left(\vec{\sigma}_{i} \cdot \vec{\sigma}_{j}\right) \sum_{a=1}^{3}\left(\lambda_{i}^{a} \cdot \lambda_{j}^{a}\right), \\
V_{\sigma}^{C}\left(\vec{r}_{i j}\right) & =-\frac{g_{c h}^{2}}{4 \pi} \frac{\Lambda_{\sigma}^{2}}{\Lambda_{\sigma}^{2}-m_{\sigma}^{2}} m_{\sigma}\left[Y\left(m_{\sigma} r_{i j}\right)-\frac{\Lambda_{\sigma}}{m_{\sigma}} Y\left(\Lambda_{\sigma} r_{i j}\right)\right], \\
V_{K}^{C}\left(\vec{r}_{i j}\right) & =\frac{g_{c h}^{2}}{4 \pi} \frac{m_{K}^{2}}{12 m_{i} m_{j}} \frac{\Lambda_{K}^{2}}{\Lambda_{K}^{2}-m_{K}^{2}} m_{K}\left[Y\left(m_{K} r_{i j}\right)-\frac{\Lambda_{K}^{3}}{m_{K}^{3}} Y\left(\Lambda_{K} r_{i j}\right)\right]\left(\vec{\sigma}_{i} \cdot \vec{\sigma}_{j}\right) \sum_{a=4}^{7}\left(\lambda_{i}^{a} \cdot \lambda_{j}^{a}\right), \\
V_{\eta}^{C}\left(\vec{r}_{i j}\right) & =\frac{g_{c h}^{2}}{4 \pi} \frac{m_{\eta}^{2}}{12 m_{i} m_{j}} \frac{\Lambda_{\eta}^{2}}{\Lambda_{\eta}^{2}-m_{\eta}^{2}} m_{\eta}\left[Y\left(m_{\eta} r_{i j}\right)-\frac{\Lambda_{\eta}^{3}}{m_{\eta}^{3}} Y\left(\Lambda_{\eta} r_{i j}\right)\right]\left(\vec{\sigma}_{i} \cdot \vec{\sigma}_{j}\right)\left[\cos \theta_{P}\left(\lambda_{i}^{8} \cdot \lambda_{j}^{8}\right)-\sin \theta_{P}\right],
\end{aligned}
$$

the angle $\theta_{P}$ appears as a consequence of considering the physical $\eta$ instead the octet one. $g_{c h}=m_{q} / f_{\pi}$, the $\lambda^{\prime} s$ are the $S U(3)$ flavor Gell-Mann matrices and the $\sigma$ 's are the spin quark Pauli matrices. $m_{i}$ is the quark mass and $m_{\pi}, m_{K}$ and $m_{\eta}$ are the masses of the $S U(3)$ Goldstone bosons, taken to be their experimental values. The $\Lambda_{i}$ 's are cutoff parameters. $m_{\sigma}$ is determined through the PCAC relation $m_{\sigma}^{2} \sim m_{\pi}^{2}+4 m_{u, d}^{2}$. Finally, $Y(x)$ is the standard Yukawa function defined by $Y(x)=e^{-x} / x$.

There are three different contributions to the tensor potential,

$$
V_{q q}^{T}\left(\vec{r}_{i j}\right)=V_{\pi}^{T}\left(\vec{r}_{i j}\right)+V_{K}^{T}\left(\vec{r}_{i j}\right)+V_{\eta}^{T}\left(\vec{r}_{i j}\right)
$$

each term given by,

$$
\begin{aligned}
V_{\pi}^{T}\left(\vec{r}_{i j}\right) & =\frac{g_{c h}^{2}}{4 \pi} \frac{m_{\pi}^{2}}{12 m_{i} m_{j}} \frac{\Lambda_{\pi}^{2}}{\Lambda_{\pi}^{2}-m_{\pi}^{2}} m_{\pi}\left[H\left(m_{\pi} r_{i j}\right)-\frac{\Lambda_{\pi}^{3}}{m_{\pi}^{3}} H\left(\Lambda_{\pi} r_{i j}\right)\right] S_{i j} \sum_{a=1}^{3}\left(\lambda_{i}^{a} \cdot \lambda_{j}^{a}\right), \\
V_{K}^{T}\left(\vec{r}_{i j}\right) & =\frac{g_{c h}^{2}}{4 \pi} \frac{m_{K}^{2}}{12 m_{i} m_{j}} \frac{\Lambda_{K}^{2}}{\Lambda_{K}^{2}-m_{K}^{2}} m_{K}\left[H\left(m_{K} r_{i j}\right)-\frac{\Lambda_{K}^{3}}{m_{K}^{3}} H\left(\Lambda_{K} r_{i j}\right)\right] S_{i j} \sum_{a=4}^{7}\left(\lambda_{i}^{a} \cdot \lambda_{j}^{a}\right), \\
V_{\eta}^{T}\left(\vec{r}_{i j}\right) & =\frac{g_{c h}^{2}}{4 \pi} \frac{m_{\eta}^{2}}{12 m_{i} m_{j}} \frac{\Lambda_{\eta}^{2}}{\Lambda_{\eta}^{2}-m_{\eta}^{2}} m_{\eta}\left[H\left(m_{\eta} r_{i j}\right)-\frac{\Lambda_{\eta}^{3}}{m_{\eta}^{3}} H\left(\Lambda_{\eta} r_{i j}\right)\right] S_{i j}\left[\cos \theta_{P}\left(\lambda_{i}^{8} \cdot \lambda_{j}^{8}\right)-\sin \theta_{P}\right],
\end{aligned}
$$


being $S_{i j}=3\left(\vec{\sigma}_{i} \cdot \hat{r}_{i j}\right)\left(\vec{\sigma}_{j} \cdot \hat{r}_{i j}\right)-\vec{\sigma}_{i} \cdot \vec{\sigma}_{j}$ the quark tensor operator and $H(x)=(1+3 / x+$ $\left.3 / x^{2}\right) Y(x)$.

Finally, the spin-orbit potential only presents a contribution coming form the scalar part of the interaction,

$$
V_{q q}^{S O}\left(\vec{r}_{i j}\right)=V_{\sigma}^{S O}\left(\vec{r}_{i j}\right)=-\frac{g_{c h}^{2}}{4 \pi} \frac{\Lambda_{\sigma}^{2}}{\Lambda_{\sigma}^{2}-m_{\sigma}^{2}} \frac{m_{\sigma}^{3}}{2 m_{i} m_{j}}\left[G\left(m_{\sigma} r_{i j}\right)-\frac{\Lambda_{\sigma}^{3}}{m_{\sigma}^{3}} G\left(\Lambda_{\sigma} r_{i j}\right)\right] \vec{L} \cdot \vec{S}
$$

where $G(x)=(1+1 / x) Y(x) / x$.

QCD perturbative effects are taken into account through the one-gluon-exchange (OGE) potential [24]. The nonrelativistic reduction of the one-gluon-exchange diagram in QCD for point-like quarks presents a contact term that, when not treated perturbatively, leads to collapse [25]. This is why one maintains the structure of the OGE, but the $\delta$ function is regularized in a suitable way. This regularization, justified by the finite size of the systems studied, has to be flavor dependent [26]. As a consequence, the central part of the OGE reads,

$$
V_{O G E}^{C}\left(\vec{r}_{i j}\right)=\frac{1}{4} \alpha_{s} \overrightarrow{\lambda c}_{i} \cdot \overrightarrow{\lambda c}_{j}\left\{\frac{1}{r_{i j}}-\frac{1}{6 m_{i} m_{j}} \vec{\sigma}_{i} \cdot \vec{\sigma}_{j} \frac{e^{-r_{i j} / r_{0}(\mu)}}{r_{i j} r_{0}^{2}(\mu)}\right\},
$$

where $\lambda^{c}$ are the $S U(3)$ color matrices, $\alpha_{s}$ is the quark-gluon coupling constant, and $r_{0}(\mu)=$ $\hat{r}_{0} \mu_{n n} / \mu_{i j}$, where $\mu_{i j}$ is the reduced mass of quarks $i j$ ( $n$ stands for the light $u$ and $d$ quarks) and $\hat{r}_{0}$ is a parameter to be determined from the data.

The noncentral terms of the OGE present a similar problem. For point-like quarks they contain an $1 / r^{3}$ term. Once again the finite size of the constituent quarks allows for a regularization, obtaining tensor and spin-orbit potentials of the form,

$$
\begin{aligned}
V_{O G E}^{T}\left(\vec{r}_{i j}\right)= & -\frac{1}{16} \frac{\alpha_{s}}{m_{i} m_{j}} \vec{\lambda}_{i}^{c} \cdot \vec{\lambda}_{j}^{c}\left[\frac{1}{r_{i j}^{3}}-\frac{e^{-r_{i j} / r_{g}(\mu)}}{r_{i j}}\left(\frac{1}{r_{i j}^{2}}+\frac{1}{3 r_{g}^{2}(\mu)}+\frac{1}{r_{i j} r_{g}(\mu)}\right)\right] S_{i j}, \\
V_{O G E}^{S O}\left(\vec{r}_{i j}\right)= & -\frac{1}{16} \frac{\alpha_{s}}{m_{i}^{2} m_{j}^{2}} \vec{\lambda}_{i}^{c} \cdot \vec{\lambda}_{j}^{c}\left[\frac{1}{r_{i j}^{3}}-\frac{e^{-r_{i j} / r_{g}(\mu)}}{r_{i j}^{3}}\left(1+\frac{r_{i j}}{r_{g}(\mu)}\right)\right] \times \\
& {\left[\left(\left(m_{i}+m_{j}\right)^{2}+2 m_{i} m_{j}\right)\left(\vec{S}_{+} \cdot \vec{L}\right)+\left(m_{j}^{2}-m_{i}^{2}\right)\left(\vec{S}_{-} \cdot \vec{L}\right)\right], }
\end{aligned}
$$

where $\vec{S}_{ \pm}=\vec{S}_{i} \pm \vec{S}_{j}$, and $r_{g}(\mu)=\hat{r}_{g} / \mu$ presents a similar behavior to the scaling of the central term.

The strong coupling constant, taken to be constant for each flavor sector, has to be scaledependent when describing different flavor sectors [27]. Such an effective scale dependence has been related to the typical momentum scale of each flavor sector assimilated to the reduced mass of the system [28]. This has been found to be relevant for the study of the meson spectra and parametrized in Ref. 21] as

$$
\alpha_{s}(\mu)=\frac{\alpha_{0}}{\ln \left[\left(\mu^{2}+\mu_{0}^{2}\right) / \gamma_{0}^{2}\right]}
$$

where $\mu$ is the reduced mass of the interacting $q q$ pair and $\alpha_{0}, \mu_{0}$, and $\gamma_{0}$ are fitted parameters.

Finally, any model imitating QCD should incorporate confinement. Lattice calculations in the quenched approximation derived, for heavy quarks, a confining interaction linearly dependent on the interquark distance. The consideration of sea quarks apart from valence 
quarks (unquenched approximation) suggests a screening effect on the potential when increasing the interquark distance [29]. Creation of light-quark pairs out of vacuum in between the quarks becomes energetically preferable resulting in a complete screening of quark color charges at large distances. String breaking has been definitively confirmed through lattice calculations [30] in coincidence with the quite rapid crossover from a linear rising to a flat potential well established in $S U(2)$ Yang-Mills theories [31]. The central part of a screened potential simulating these results can be written as,

$$
V_{C O N}^{C}\left(\vec{r}_{i j}\right)=-a_{c}\left(1-e^{-\mu_{c} r_{i j}}\right)\left(\vec{\lambda}_{i} \cdot \overrightarrow{\lambda c}_{j}\right)
$$

At short distances it presents a linear behavior with an effective confinement strength $a=$ $a_{c} \mu_{c} \overrightarrow{\lambda c}_{i} \cdot \overrightarrow{\lambda c}_{j}$, while it becomes constant at large distances. Screened confining potentials have been analyzed in the literature providing an explanation to the missing state problem in the baryon spectra [32], improving the description of the heavy-meson spectra [33], and justifying the deviation of the meson Regge trajectories from the linear behavior for higher angular momentum states [34].

Confinement also presents an spin-orbit contribution taken to be an arbitrary combination of scalar and vector terms of the form

$$
\begin{aligned}
V_{C O N}^{S O}\left(\vec{r}_{i j}\right)= & -\left(\vec{\lambda}_{i}^{c} \cdot \vec{\lambda}_{j}^{c}\right) \frac{a_{c} \mu_{c} e^{-\mu_{c} r_{i j}}}{4 m_{i}^{2} m_{j}^{2} r_{i j}}\left[\left(\left(m_{i}^{2}+m_{j}^{2}\right)\left(1-2 a_{s}\right)\right.\right. \\
& \left.\left.+4 m_{i} m_{j}\left(1-a_{s}\right)\right)\left(\vec{S}_{+} \cdot \vec{L}\right)+\left(m_{j}^{2}-m_{i}^{2}\right)\left(1-2 a_{s}\right)\left(\vec{S}_{-} \cdot \vec{L}\right)\right]
\end{aligned}
$$

where $a_{s}$ would control the ratio between them.

Once perturbative (one-gluon exchange) and nonperturbative (confinement and chiral symmetry breaking) aspects of QCD have been considered, one ends up with a quark-quark interaction of the form

$$
V_{q_{i} q_{j}}=\left\{\begin{array}{l}
q_{i} q_{j}=n n / s n \Rightarrow V_{C O N}+V_{O G E}+V_{\chi} \\
q_{i} q_{j}=c n / c s \Rightarrow V_{C O N}+V_{O G E}
\end{array} .\right.
$$

Note that for the particular case of heavy quarks, chiral symmetry is explicitly broken and therefore Goldstone boson exchanges do not contribute. The model parameters and a more detailed discussion of the model can be found in Refs. [20, 21, 23].

\section{RESULTS AND DISCUSSION}

A thoroughly study of the full meson spectra has been presented in Ref. [21]. The results for the open-charm mesons are resumed in Table II It can be seen how the open-charm states are easily identified with standard $c \bar{q}$ mesons except for the cases of the $D_{s J}^{*}(2317)$, the $D_{s J}(2460)$, and the $D_{0}^{*}(2308)$. We will also comment on the recent measurement of the $D_{1}^{0}(2430)$. This behavior is shared by almost all quark potential model calculations [4]. Although the situation from lattice QCD is far from being definitively established, similar problems are observed. Lattice NRQCD in the quenched approximation predicts for the $D_{s J}^{*}(2317)$ a mass of $2.44 \mathrm{GeV}$ [36], while using relativistic charm quarks the mass obtained is $2.47 \mathrm{GeV}$ 37]. Unquenched lattice QCD calculations of $c \bar{s}$ states do not find a window for the $D_{s J}^{*}(2317)[19]$, supporting the difficulty of a $P$-wave $c \bar{s}$ interpretation. The quenched lattice QCD calculation of the spectrum of orbitally excited $D_{s}$ mesons of Ref. [38] concludes 
that, although the results obtained are consistent with a $c \bar{s}$ configuration, the statistical and systematical uncertainties are too large to exclude the exotic states based on potential quark models [4]. The same situation may be drawn from heavy quark symmetry arguments. One finds that the scalar $c \bar{s}$ state belongs to the $j=1 / 2$ doublet, but since the $j=3 / 2$ doublet is identified with the narrow $D_{s 2}(2573)$ and $D_{s 1}(2536)$ (with total widths of $15_{-4}^{+5} \mathrm{MeV}$ and $<2.3 \mathrm{MeV}$, respectively) the scalar state is expected to have a much larger width than the one measured for the $D_{s J}^{*}(2317)$ [39].

Thus, one could be tempted to interpret these states as four-quark resonances within the quark model. The results obtained for the $c n \bar{s} \bar{n}$ and $c n \bar{n} \bar{n}$ configurations with the same interacting potential of Sec. II are shown in Table II The $I=1$ and $I=0$ states are far above the corresponding strong decay threshold and therefore should be broad, what rules out a pure four-quark interpretation of the new open-charm mesons.

As outlined above, for $P$-wave mesons the hadronic dressing is in a $S$-wave, thus physical states may correspond to a mixing of two- and four-body configurations, Eq. (11). In the isoscalar sector, the $c n \bar{s} \bar{n}$ and $c \bar{s}$ states get mixed, as it happens with $c n \bar{n} \bar{n}$ and $c \bar{n}$ for the $I=1 / 2$ case. Let us notice that the interacting potential contains terms mixing $P$-wave $S=0$ and $S=1$ states [21], arising from the different spin-orbit contributions for systems made of quarks of different mass. The mixing angle comes determined by the structure of the interaction. The parameter $\gamma$ has been fixed to reproduce the mass of the $D_{s J}^{*}(2317)$ meson, being $\gamma=240 \mathrm{MeV}$. Using this value one has $\left|\gamma /\left(E_{J P C}^{n}-E_{J P C}^{n+1}\right)\right|^{2} \approx 0.25 \ll 1$, a ratio consistent with the assumption that the hamiltonian $H_{1}$, Eq. (2), can be treated perturbatively. This perturbative condition together with $\left|\gamma / E_{q \bar{q}}\right| \approx\left|\gamma / E_{q q \bar{q} \bar{q}}\right| \approx 0.1 \ll 1$ also ensures that the mixing with higher states can be neglected.

The results obtained are shown in Table III. Let us first analyze the nonstrange sector. The ${ }^{3} P_{0} c \bar{n}$ pair and the $c n \bar{n} \bar{n}$ have a mass of $2465 \mathrm{MeV}$ and $2505 \mathrm{MeV}$, respectively. Once the mixing is considered one obtains a state at $2241 \mathrm{MeV}$ with $46 \%$ of four-quark component and $53 \%$ of $c \bar{n}$ pair. The lowest state, representing the $D_{0}^{*}(2308)$, is above the isospin preserving threshold $D \pi$, being broad as observed experimentally. The mixed configuration compares much better with the experimental data than the pure $c \bar{n}$ state. The orthogonal state appears higher in energy, at $2713 \mathrm{MeV}$, with and important four-quark component. A similar process would modify the state representing the $D_{1}^{0}(2430)$, but in this case one would need the mass of the $I=1 / 2 J^{P}=1^{+}$four-quark state. The huge basis generated for such quantum numbers makes unfeasible its calculation. A correct description of this state would require a mass of $2.9 \mathrm{GeV}$ for the four quark state mentioned above.

Concerning the strange sector, the $D_{s J}^{*}(2317)$ and the $D_{s J}(2460)$ are dominantly $c \bar{s} J=$ $0^{+}$and $J=1^{+}$states, respectively, with almost $30 \%$ of four-quark component. Without being dominant, it is fundamental to shift the mass of the unmixed states to the experimental values below the $D K$ and $D^{*} K$ thresholds. Being both states below their isospin-preserving two-meson threshold, the only allowed strong decays to $D_{s}^{*} \pi$ would violate isospin and are expected to have small widths. This width has been estimated assuming either a $q \bar{q}$ structure [12, 40], a four-quark state [41] or vector meson dominance [42] obtaining in all cases a width of the order of $10 \mathrm{keV}$. The second isoscalar $J^{P}=1^{+}$state, with an energy of $2555 \mathrm{MeV}$ and $98 \%$ of $c \bar{s}$ component, corresponds to the $D_{s 1}(2536)$. Regarding the $D_{s J}^{*}(2317)$, it has been argued that a possible $D K$ molecule would be preferred with respect to an $I=0 \mathrm{cn} \bar{s} \bar{n}$ tetraquark, what would anticipate an $I=1 \mathrm{cn} \bar{s} \bar{n}$ partner nearby in mass [14]. Our results confirm the last argument, the vicinity of the isoscalar and isovector tetraquarks (see Table II), however, the restricted coupling to the $c \bar{s}$ system allowed only for the $I=0$ four-quark 
states opens the possibility of a mixed nature for the $D_{s J}^{*}(2317)$, the $I=1$ tetraquark partner remaining much higher in energy. The $I=1 J=0^{+}$and $J=1^{+}$four-quark states appear above $2700 \mathrm{MeV}$ and cannot be shifted to lower energies.

Our results do not show any state around $2600 \mathrm{MeV}$, the mass region compatible with the $D_{s J}^{+}(2632)$ measured by SELEX. Its two possible theoretical partners, the $2^{3} S_{1}$ state (suggested in Ref. 9]) or the $1^{3} D_{1}$ state, lie above $2700 \mathrm{MeV}$ (2764 and $2873 \mathrm{MeV}$, respectively). This state has been proposed as the $c s \bar{s} \bar{s}$ partner of the $D_{s J}^{*}(2317)$ [17]. Naively, the $D_{s J}^{*}(2317)$ sets a scale of $2320 \mathrm{MeV}$ for the $c n \bar{s} \bar{n}$ sector, and an augment of $150 \mathrm{MeV}$ for each strange quark could accommodate a $c s \bar{s} \bar{s}$ system near the mass of the $D_{s J}^{+}(2632)$. However, the mass of the $D_{s J}^{*}(2317)$ is obtained through the coupling to the $c \bar{s}$ quark pair, being the mass of the tetraquark configuration above $2700 \mathrm{MeV}$, disregarding a possible $c s \bar{s} \bar{s}$ interpretation of the $D_{s J}^{+}(2632)$. It has also been discarded as the first radial excitation of the $D_{s}^{*}(2112)$ [43]. A careful analysis of several theoretical interpretations has been done in Ref. [44], the surprising properties of this state not fitting in any of the scenarios considered. Several experiments have failed trying to confirm the existence of this state 8]. The confirmation or refutation of the $D_{s J}^{+}(2632)$ is clearly an important priority for meson spectroscopy.

The structure of the $D_{s J}^{*}(2317)$ and the $D_{s J}(2460)$ mesons could be scrutiny, apart from their masses, also through the study of their electromagnetic decay widths. Using the standard formalism described, for example, in Ref. [40], the $E 1$ radiative transitions for $c \bar{s} \rightarrow c \bar{s}+\gamma$ are given by:

$$
\Gamma[c \bar{s} \rightarrow c \bar{s}+\gamma]=\frac{4}{27} \alpha\left\langle e_{Q}\right\rangle^{2} \omega^{3}\left(2 J_{f}+1\right)\left|\left\langle{ }^{2 S+1} S_{J^{\prime}}|r|^{2 S+1} P_{J}\right\rangle\right|^{2} S_{i f}
$$

where $S_{i f}$ is a statistical factor with $S_{i f}=1$ for the transitions between spin-triplet states and $S_{i f}=3$ for the transition between spin-singlet states, $\left\langle e_{Q}\right\rangle$ is an effective quark charge given by

$$
\left\langle e_{Q}\right\rangle=\frac{m_{s} e_{c}-m_{c} e_{\bar{s}}}{m_{c}+m_{s}}
$$

where $e_{i}$ is the charge of the quark(antiquark) $i$ in units of $|e|, \alpha$ is the fine structure constant and $\omega$ is the photon energy. Once the mixing between two- and four-quark components has been included there would also be a contribution to the decay width coming from $c n \bar{s} \bar{n} \rightarrow c \bar{s}+\gamma$, that goes necessarily through the annihilation of a color singlet $n \bar{n}$ pair with photon quantum numbers within the four-quark wave function. The contribution to the electromagnetic decay width arising from the four-quark component gets suppressed. This can be illustrated by the analysis of the $M 1$ electromagnetic decay width for $c n \bar{s} \bar{n}\left(1^{+}\right) \rightarrow$ $c n \bar{s} \bar{n}\left(0^{+}\right)+\gamma$, that is given by

$$
\Gamma\left[c n \bar{s} \bar{n}\left(1^{+}\right) \rightarrow c n \bar{s} \bar{n}\left(0^{+}\right)+\gamma\right]=\frac{4}{3} \alpha \omega^{3}\left(2 J_{f}+1\right)\left|\left\langle{ }^{1} S_{0}\left|\mu_{z}\right|^{3} S_{1}\right\rangle\right|^{2}
$$

where

$$
\mu_{z}=\sum_{i=1}^{4} \frac{e_{i}}{2 m_{i}} \sigma_{i z} .
$$

From the above equation one obtains $\Gamma\left[c n \bar{s} \bar{n}\left(1^{+}\right) \rightarrow c n \bar{s} \bar{n}\left(0^{+}\right)+\gamma\right] \approx 4 \mathrm{eV}$, that will contribute to the $\Gamma\left[D_{s J}(2460) \rightarrow D_{s J}^{*}(2317)+\gamma\right]$ decay width, once the configuration probabilities given in Table III are considered, in the order of $0.25 \mathrm{eV}$. The value obtained in Ref. 
[40] for this decay, assuming a pure $c \bar{s}$ configuration, is one order of magnitude larger confirming the smallness of the four to four-quark contribution. In Ref. [45] this decay width was estimated assuming a molecular structure, obtaining a larger value of the order of 17 $\mathrm{keV}$. This makes evident the important difference between the assumed molecular structure of Ref. [45] and the full multiquark configuration considered in this work. In the case of the four-quark configuration the orthogonality between the several components of the wave functions diminishes the decay widths, an effect that does not seem to be present within the molecular interpretation. The same reasoning applies to the $\Gamma[c n \bar{s} \bar{n} \rightarrow c \bar{s}+\gamma]$ decay width, being suppressed due to the reduced singlet-singlet component with photon quantum numbers within the four-quark wave function. Similar conclusions were obtained in Ref. 18]. As a consequence, the presence of a four-quark component diminishes the decay widths, making them different from those predicted for a pure $c \bar{s}$ state [14, 40, 42].

We compare in Table [IV our results for the radiative transitions of the $D_{s J}^{*}(2317)$ and $D_{s J}(2460)$ with different theoretical approaches and the experimental limits reported by CLEO and Belle. The main difference is noticed in the suppression predicted for the $D_{s J}(2460) \rightarrow D_{s}^{*+} \gamma$ decay as compared to the $D_{s J}(2460) \rightarrow D_{s}^{+} \gamma$. A ratio $D_{s J}(2460) \rightarrow D_{s}^{+} \gamma / D_{s J}(2460) \rightarrow D_{s}^{*+} \gamma \approx 1-2$ has been obtained assuming a $q \bar{q}$ structure for both states [12, 40] (what seems incompatible with their properties). Heavy-hadron chiral perturbation theory calculations find a similar ratio [46]. We find a larger value, $D_{s J}(2460) \rightarrow D_{s}^{+} \gamma / D_{s J}(2460) \rightarrow D_{s}^{*+} \gamma \approx 100$, due to the small $1^{3} P_{1} c \bar{s}$ probability of the $D_{s J}(2460)$. A similar enhancement has been obtained in Ref. [47] (see penultimate column of Table IV] in the framework of light-cone QCD sum rules in contrast to a previous calculation of the same authors using vector meson dominance [42]. As a consequence, the radiative transitions are an important diagnostic tool to understand the nature of these states. In view of the different predictions of the electromagnetic decay widths, a precise measurement of this decay would allow to distinguish not only between $q \bar{q}$ and non $-q \bar{q}$ states, but also between pure molecular and four-quark interpretations.

Let us finally mention that the difficulties encountered for the interpretation of the new open-charm states as two-quark systems do not appear for the case of the recent charmed and $B_{c}$ states measured at different facilities. They do nicely fit into the predictions of the model used, see Table $\nabla$ giving confidence to the results obtained in the present work.

The interpretation we have just presented of the positive parity open-charmed mesons as a mixture of two- and four-quark states has also been used to account for the experimentally observed light-scalar mesons within the same constituent quark model [20], what gives us confidence on the mechanism proposed. Nonetheless, one should not forget that in the literature there is a wide variety of interpretations for the open-charm and also for the light-scalar mesons. As previously mentioned, they used to deal with one of both problems and even then with a particular set of states, being the most common ones the strange open-charm states and the isoscalar light mesons.

Regarding the light-scalar mesons, there have been reported by the PDG two isovectors, five isoscalar and three isodoublet states. The situation is far from being definitively settled, neither from the experimental nor from the theoretical point of view. Experimentally, let us only mention that the recent analysis by BES of the $J / \Psi \rightarrow \phi \pi^{+} \pi^{-}$and $J / \Psi \rightarrow \phi K^{+} K^{-}$ data [53] requires a state $f_{0}(1790)$ distinct from the $f_{0}(1710)$. Recent reanalysis of the Crystal Barrel data [54] suggest the existence of a new state called $f_{0}(1200-1600)$. Theoretically, one would expect non $-q \bar{q}$ scalar objects in the mass range below $2 \mathrm{GeV}$. Multiquarks have been justified to coexist with $q \bar{q}$ states in the energy region around $1 \mathrm{GeV}$ because they can 
couple to $0^{++}$without orbital excitation [55]. Lattice QCD in the quenched approximation predicts the existence of a scalar glueball with a mass around $1.6 \mathrm{GeV}[56]$.

From this complicated scenario many different interpretations of the light-scalar mesons have arisen. The overpopulation of $0^{++}$states below $2 \mathrm{GeV}$ gave rise long ago to the speculation of the existence of four light-quark states. The most complete analysis was performed by Weinstein and Isgur [26, 57], concluding that, normally, $q q \bar{q} \bar{q}$ bound states do not exist, being the only exception the scalar sector where weakly bound states with a mesonmeson molecule structure were found. Particular sets of states have been studied in the literature. There are several models analyzing the mixing between different configurations to yield the physical $f_{0}(1370), f_{0}(1500)$, and $f_{0}(1710)$. Among them, Ref. 20] assigning the larger glueball component to the $f_{0}(1710)$ is on the line with Refs. [58] and differ from those of Refs. [59, 60] concluding that the $f_{0}(1710)$ is dominantly $q \bar{q}$. One should notice that in these studies only Refs. [20, 60] consider the recently reported $f_{0}(1790)$. In a different fashion within the quark model, the $a_{0}(980)$ and $f_{0}(980)$ mesons were analyzed in Ref. 61], being the effect of the two-pseudoscalar meson thresholds the responsible for the substantial shift to a lower mass than what is naively expected from the $q \bar{q}$ component alone. This gives rise to an important $K \bar{K}$ and $\pi \eta^{\prime}$ components in the $a_{0}(980)$ and $K \bar{K}, \eta \eta, \eta^{\prime} \eta^{\prime}$ and $\eta \eta^{\prime}$ in the $f_{0}(980)$.

The structure of the scalar mesons $a_{0}(980)$ and $f_{0}(980)$ have been also investigated in the framework of a meson exchange based on the Jülich potential model [62] for $\pi \pi$ and $\pi \eta$ scattering. Whereas the $f_{0}(980)$ appears to be a $K \bar{K}$ bound state the $a_{0}(980)$ was found to be a dynamically generated threshold effect. Similar conclusions have been obtained in a chiral unitary coupled channel approach, where the $f_{0}(600)$, the $a_{0}(980)$, and the $K_{0}^{*}(800)$ rise up as dynamically generated resonances, while the $f_{0}(980)$ is a combination of a strong $S$-wave meson-meson unitarity effect and a preexisting singlet resonance [63]. In Ref. 64] van Beveren et al. describe the light scalar mesons as resonances and bound states characterized by complex singularities of the scattering amplitude.

Finally, let us stress that Ref. [20] presents an interpretation of the scalar mesons in a model constrained by the description of other hadron sectors. The same mechanism has been applied here to disentangle the structure of the new $D_{s}$ and $D_{s J}$ resonances. It drives to a final scenario that it is compatible with some other models in the literature and it differs from other results. Being the set of data so huge, and sometimes so poor, one always may find a positive or negative interpretation of some of them. Therefore, the final answer could only be obtained from precise experimental data that would allow to discriminate between the predictions of different theoretical models [65].

\section{SUMMARY}

As a summary, we have obtained a rather satisfactory description of the positive parity open-charm mesons in terms of two- and four-quark configurations. The mixing between these two components is responsible for the unexpected low mass and widths of the $D_{s J}^{*}(2317), D_{s J}(2460)$, and $D_{0}^{*}(2308)$. The same mechanism has been used to account for the spectroscopic properties of the light-scalar mesons. The obtained electromagnetic decay widths give hints that would help in distinguishing the nature of these states. We predict a ratio $D_{s J}(2460) \rightarrow D_{s}^{+} \gamma / D_{s J}(2460) \rightarrow D_{s}^{*+} \gamma$ much larger than the one obtained in a pure $q \bar{q}$ scheme. We did not find any theoretical partner for the recently measured $D_{s J}^{+}(2632)$ whose existence awaits confirmation [8]. We encourage experimentalists on two different 
directions: the measurement of the electromagnetic decay widths of the $D_{s J}^{*}(2317)$ and the $D_{s J}(2460)$, and the confirmation or refutation of the $D_{s J}^{+}(2632)$ that would help to clarify the exciting situation of the open-charm mesons.

\section{ACKNOWLEDGMENTS}

This work has been partially funded by Ministerio de Ciencia y Tecnología under Contract No. FPA2004-05616, by Junta de Castilla y León under Contract No. SA-104/04, and by Generalitat Valenciana under Contract No. GV05/276.

[1] BABAR Collaboration, B. Aubert et al., Phys. Rev. Lett. 90, 242001 (2003).

[2] CLEO Collaboration, D. Besson et al., Phys. Rev. D 68, 032002 (2003).

[3] Belle Collaboration, Y. Mikani et al., Phys. Rev. Lett. 92, 012002 (2004).

[4] S. Godfrey and R. Kokoski, Phys. Rev. D 43, 1679 (1991). D. Ebert, V.O. Galkin, and R.N. Faustov, Phys. Rev. D 57, 5663 (1998). M. Di Pierro and E. Eichten, Phys. Rev. D 64, 114004 (2001). W. Lucha and F.F. Schöberl, Mod. Phys. Lett A 18, 2837 (2003). S. Godfrey, J. Phys. Conf. Ser. 9, 59 (2005).

[5] Belle Collaboration, K. Abe et al., Phys. Rev. D 69, 112002 (2004).

[6] FOCUS Collaboration, J.M. Link et al., Phys. Lett. B 586, 11 (2004).

[7] SELEX Collaboration, A.V. Evdokimov et al., Phys. Rev. Lett. 93, 242001 (2004).

[8] BABAR Collaboration, B. Aubert et al., hep-ex/0408087. Belle Collaboration, B. Yabsley, hep-ex/0507028. FOCUS Collaboration, R. Kutschke, E831-doc-701-v2.

[9] E. van Beveren and G. Rupp, Phys. Rev. Lett. 91, 012003 (2003).

[10] Yu.A. Simonov and J.A. Tjon, Phys. Rev. D 70, 114013 (2004).

[11] R.N. Cahn and J.D. Jackson, Phys. Rev. D 68, 037502 (2003).

[12] W.A. Bardeen, E.J. Eichten, and C.T. Hill, Phys. Rev. D 68, 054024 (2003).

[13] D.S. Hwang and D.W. Kim, Phys. Lett. B 601, 137 (2004).

[14] T. Barnes, F.E. Close, and H.J. Lipkin, Phys. Rev. D 68, 054006 (2003).

[15] A.P. Szczepaniak, Phys. Lett. B 567, 23 (2003). K. Terasaki, Phys. Rev. D 68, 011501(R) (2003).

[16] H.Y. Cheng and W.S. Hou, Phys. Lett. B 566, 193 (2003).

[17] Y.-Q. Chen and X.-Q. Li, Phys. Rev. Lett. 93, 232001 (2003).

[18] T. Browder, S. Pakvasa, and A. Petrov, Phys. Lett. B 578, 365 (2004).

[19] G.S. Bali, Phys. Rev. D 68, 071501(R) (2003).

[20] J. Vijande, A. Valcarce, F. Fernández, and B. Silvestre-Brac, Phys. Rev. D 72, 034025 (2005).

[21] J. Vijande, F. Fernández, and A. Valcarce, J. Phys. G 31, 481 (2005).

[22] Y. Suzuki and K. Varga, Lecture Notes in Physics M 54, 1 (1998).

[23] J. Vijande, F. Fernández, A. Valcarce, and B. Silvestre-Brac, Eur. Phys. J. A 19383 (2004).

[24] A. de Rújula, H. Georgi, and S.L. Glashow, Phys. Rev. D 12, 147 (1975).

[25] R.K. Bhaduri, L.E. Cohler, and Y. Nogami, Phys. Rev. Lett. 44, 1369 (1980).

[26] J. Weinstein and N. Isgur, Phys. Rev. D 27, 588 (1983).

[27] S. Titard and F.J. Yndurain, Phys. Rev. D 51, 6348 (1995). A.M. Badalian and B.L.G. Bakker, Phys. Rev. D 62, 094031 (2000). 
[28] F. Halzen, C. Olson, M.G. Olsson and M.L. Stong, Phys. Rev. D 47, 3013 (1993).

[29] G.S. Bali, Phys. Rep. 343, 1 (2001).

[30] SESAM Collaboration, G.S. Bali, H. Neff, T. Düssel, T. Lippert, and K. Schilling, Phys. Rev. D 71, 114513 (2005).

[31] P.W. Stephenson, Nucl. Phys. B 550, 427 (1999). P. de Forcrand and O. Philipsen, Phys. Lett. B 475, 280 (2000).

[32] J. Vijande, P. González, H. Garcilazo, and A. Valcarce, Phys. Rev. D 69, 074019 (2004).

[33] P. González, A. Valcarce, H. Garcilazo, and J. Vijande, Phys. Rev. D 68, 034007 (2003).

[34] M.M. Brisudová, L. Burakovsky, and T. Goldman, Phys. Rev. D 61, 054013 (2000).

[35] S. Eidelman et al., Phys. Lett. B 592, 1 (2004).

[36] J. Hein et al., Phys. Rev. D 62, 074503 (2000).

[37] UKQCD Collaboration, P. Boyle, Nucl. Phys. B (Proc. Supp.) 63, 314 (1998); et al., Nucl. Phys. B (Proc. Supp.) 53, 398 (1997).

[38] UKQCD Collaboration, A. Dougall, et al. Phys. Lett. B 569, 41 (2003).

[39] N. Isgur and M. B. Wise, Phys. Lett. B 237, 527 (1990); ibid Phys. Lett. B 232, 113 (1989).

[40] S. Godfrey, Phys. Lett. B 568, 254 (2003).

[41] M. Nielsen, hep-ph/0510277.

[42] P. Colangelo and F. de Fazio, Phys. Lett. B 570, 180 (2003). P. Colangelo and F. de Fazio, and R. Ferrandes, Mod. Phys. Lett. A 19, 2083 (2004).

[43] C. Chang, C.S. Kim, and G. Wang, Phys. Lett. B 623, 218 (2005).

[44] T. Barnes, F.E. Close, J.J. Dudek, S. Godfrey, and E.S. Swanson, Phys. Lett. B 600, 223 (2004).

[45] F. E. Close and E. S. Swanson, Phys. Rev. D 72, 094004 (2005).

[46] T. Mehen and R.P. Springer, Phys. Rev. D 70, 074014 (2000).

[47] P. Colangelo, F. De Fazio, and A. Ozpineci, Phys. Rev. D 72, 074004 (2005).

[48] Belle Collaboration, K. Abe et al., hep-ex/0507019.

[49] Belle Collaboration, S.-K. Choi et al., Phys. Rev. Lett. 94, 182002 (2005).

[50] Belle Collaboration, K. Abe et al., hep-ex/0512035

[51] BABAR Collaboration, B. Aubert et al., Phys. Rev. Lett. 95, 142001 (2005).

[52] CDF Collaboration, M.D. Corcoran, hep-ex/0506061

[53] BES Collaboration, M. Ablikim, et al., Phys. Lett. B 607, 243 (2005).

[54] V.V. Anisovich and A.V. Sarantsev, Eur. Phys. J. A 16, 229 (2003).

[55] R.L. Jaffe, Phys. Rev. D 15, 267 (1977).

[56] C.J. Morningstar and M. Peardon, Phys. Rev. D 60, 034509 (1999).

[57] J. Weinstein and N. Isgur, Phys. Rev. Lett. 48, 659 (1982); ibid Phys. Rev. D 41, 2236 (1990).

[58] C. McNeile and C. Michael, Phys. Rev. D 63, 114503 (2001); W. Lee and D. Weingarten, Phys. Rev. D 61, 014015 (2000); D.-M. Li, H. Yu, and Q.-X. Shen, Commun. Theor. Phys. 34, 507 (2000); L.S. Celenza et al., Phys. Rev. C 61, 035201 (2000).

[59] C. Amsler, Phys. Lett. B 541, 22 (2002); C. Amsler and F.E. Close, Phys. Rev. D 53, 295 (1996); F.E. Close and A. Kirk, Eur. Phys. J. C 21, 531 (2001).

[60] F.E. Close and Q. Zhao, Phys. Rev. D 71, 094022 (2005).

[61] N.A. Tornqvist, Z. Phys. C 68, 647 (1995).

[62] G. Janssen, B.C. Pearce, K. Holinde and J. Speth, Phys. Rev. D 52, 2690 (1995).

[63] J.A. Oller and E. Oset, Phys. Rev. D 60, 074023 (1999). J.A. Oller, E. Oset and J.R. Peláez, Phys. Rev. Lett. 80, 3452 (1998).

[64] E. van Beveren, et al., Z. Phys. C 30, 615 (1986); E. van Beveren and G. Rupp, Eur. Phys. 
J. C 10, 469 (1999).

[65] C. Amsler and N.A. Tornqvist, Phys. Rep. 389, 61 (2004) and references therein. 
TABLE I: $c \bar{s}$ and $c \bar{n}$ masses (QM), in MeV. Experimental data (Exp.) are taken from Ref. 35], except for the state denoted by a dagger that has been taken from Ref. [5].

\begin{tabular}{|c|c|c|c|c|c|c|}
\hline$\overline{n L J^{P}}$ & State & $\mathrm{QM}(c \bar{s})$ & Exp. & State & $\mathrm{QM}(c \bar{n})$ & Exp. \\
\hline $1 S 0^{-}$ & $D_{s}$ & 1981 & $1968.5 \pm 0.6$ & $D$ & 1883 & $1867.7 \pm 0.5$ \\
\hline $1 S 1^{-}$ & $D_{s}^{*}$ & 2112 & $2112.4 \pm 0.7$ & $D^{*}$ & 2010 & $2008.9 \pm 0.5$ \\
\hline $1 P 0^{+}$ & $D_{s J}^{*}(2317)$ & 2489 & $2317.4 \pm 0.9$ & $D_{0}^{*}(2308)$ & 2465 & $2308 \pm 17 \pm 15 \pm 28$ \\
\hline $1 P 1^{+}$ & $D_{s J}(2460)$ & 2578 & $2459.3 \pm 1.3$ & $D_{1}(2420)$ & 2450 & $2422.2 \pm 1.8$ \\
\hline $1 P 1^{+}$ & $D_{s 1}(2536)$ & 2543 & $2535.3 \pm 0.6$ & $D_{1}^{0}(2430)$ & 2546 & $2427 \pm 26 \pm 25$ \\
\hline $1 P 2^{+}$ & $D_{s 2}(2573)$ & 2582 & $2572.4 \pm 1.5$ & $D_{2}^{*}(2460)$ & 2496 & $2459 \pm 4$ \\
\hline
\end{tabular}

TABLE II: $c n \bar{s} \bar{n}$ and $c n \bar{n} \bar{n}$ masses, in $\mathrm{MeV}$.

\begin{tabular}{|c|c|c|}
\hline \multicolumn{2}{|c|}{$c n \bar{s} \bar{n}$} & $c n \bar{n} \bar{n}$ \\
\hline$J^{P}=0^{+}$ & $J^{P}=1^{+}$ & $J^{P}=0^{+}$ \\
$I=0 \quad I=1$ & $I=0 \quad I=1$ & $I=1 / 2$ \\
\hline $2731 \quad 2699$ & $2841 \quad 2793$ & 2505 \\
\hline
\end{tabular}

TABLE III: Probabilities (P), in \%, of the wave function components and masses (QM), in MeV, of the open-charm mesons once the mixing between $q \bar{q}$ and $q q \bar{q} \bar{q}$ configurations is considered. Experimental data (Exp.) are taken from Ref. [35] except for the state denoted by a dagger that has been taken from Ref. [5].

\begin{tabular}{|c|cc||c|cc||c|cc|}
\hline \multicolumn{7}{|c||}{$I=0$} & \multicolumn{3}{c|}{$I=1 / 2$} \\
\hline \multicolumn{3}{|c||}{$J^{P}=0^{+}$} & \multicolumn{3}{|c||}{$J^{P}=1^{+}$} & \multicolumn{3}{c|}{$J^{P}=0^{+}$} \\
\hline $\mathrm{QM}$ & 2339 & 2847 & $\mathrm{QM}$ & 2421 & 2555 & $\mathrm{QM}$ & 2241 & 2713 \\
Exp. & $2317.4 \pm 0.9$ & - & Exp. & $2459.3 \pm 1.3$ & $2535.3 \pm 0.6$ & Exp. & $2308 \pm 17 \pm 15 \pm 28^{\dagger}$ & - \\
\hline $\mathrm{P}(c n \bar{s} \bar{n})$ & 28 & 55 & $\mathrm{P}(c n \bar{s} \bar{n})$ & 25 & $\sim 1$ & $\mathrm{P}(c n \bar{n} \bar{n})$ & 46 & 49 \\
$\mathrm{P}\left(c \bar{s}_{1^{3} P}\right)$ & 71 & 25 & $\mathrm{P}\left(c \bar{s}_{1^{1} P}\right)$ & 74 & $\sim 1$ & $\mathrm{P}\left(c \bar{n}_{1 P}\right)$ & 53 & 46 \\
$\mathrm{P}\left(c \bar{s}_{2^{3} P}\right)$ & $\sim 1$ & 20 & $\mathrm{P}\left(c \bar{s}_{1^{3} P}\right)$ & $\sim 1$ & 98 & $\mathrm{P}\left(c \bar{n}_{2 P}\right)$ & $\sim 1$ & 5 \\
\hline
\end{tabular}


TABLE IV: Comparison of 90\% C.L. limits on radiative transitions obtained by CLEO [2] and Belle 3] with our results (QM) and those of two different quark models, Refs. 12, 40], based only on $q \bar{q}$ components. The BR's are with respect to the decay $D_{s J}^{*}(2317) \rightarrow D_{s}^{+} \pi^{0}$ for the $D_{s J}^{*}(2317)$ and with respect to the decay $D_{s J}(2460) \rightarrow D_{s}^{*+} \pi^{0}$ for the $D_{s J}(2460)$. We have assumed $\Gamma\left(D_{s J}^{*}(2317) \rightarrow D_{s}^{+} \pi^{0}\right) \approx \Gamma\left(D_{s J}(2460) \rightarrow D_{s}^{*+} \pi^{0}\right) \approx 10 \mathrm{keV}$ as explained in the text. We have also quoted results obtained by light-cone QCD sum rules [47] and vector meson dominance [42].

\begin{tabular}{|c|ccc|cc|cc|}
\hline & \multicolumn{3}{|c|}{ Quark models } & \multicolumn{2}{c|}{ Experiments } & \multicolumn{2}{c|}{ Other approaches } \\
Transition & QM & Ref. [12] & Ref. [40] & CLEO [2] & Belle [3] & Ref. [47] & Ref. [42] \\
\hline$D_{s J}^{*}(2317) \rightarrow D_{s}^{*+} \gamma$ & 0.16 & 0.17 & 0.19 & $<0.059$ & $<0.18$ & $0.4-0.6$ & 0.085 \\
$D_{s J}^{*}(2317) \rightarrow D_{s}^{+} \gamma$ & 0.0 & 0.0 & 0.0 & $<0.052$ & $<0.05$ & 0.0 & 0.0 \\
$D_{s J}(2460) \rightarrow D_{s}^{*+} \gamma$ & 0.006 & 0.47 & 0.55 & $<0.16$ & $<0.31$ & $0.06-0.11$ & 0.15 \\
$D_{s J}(2460) \rightarrow D_{s}^{+} \gamma$ & 0.67 & 0.51 & 0.62 & $<0.49$ & $0.55 \pm 0.13 \pm 0.08$ & $1.9-2.9$ & 0.33 \\
\hline
\end{tabular}

TABLE V: Masses (QM), in MeV, of the recently measured charmonium and $B_{c}$ states obtained within the model of Ref. 21] used in this work.

\begin{tabular}{|ccr|cr|}
\hline Name & Mass & Ref. & $n^{2 S+1} L_{J}$ & $\mathrm{QM}$ \\
\hline$X(3940)$ & $3943 \pm 6 \pm 6$ & {$[48]$} & $2^{1} P_{1}$ & 3923 \\
- & - & & $2^{3} P_{0}$ & 3878 \\
$Y(3940)$ & $3943 \pm 11 \pm 13$ & {$[49]$} & $2^{3} P_{1}$ & 3915 \\
$X_{c 2}^{\prime}(3940)$ & $3931 \pm 4 \pm 2$ & {$[50]$} & $2^{3} P_{2}$ & 3936 \\
$Y(4260)$ & $4260 \pm 8 \pm 2$ & {$[51]$} & $4^{3} S_{1}$ & 4307 \\
$B_{c}(6287)$ & $6287 \pm 4.8 \pm 1.1$ & {$[52]$} & $1^{1} S_{0}$ & 6277 \\
\hline
\end{tabular}

